\title{
Incidencia del delirium postoperatorio en pacientes que ingresan a la Unidad de Terapia Intensiva (UTI) del Hospital Ángeles Pedregal
}

\author{
Incidence of postoperative delirium in patients admitted to the \\ Intensive Care Unit (ICU) of Hospital Ángeles Pedregal
}

\author{
Eduardo Teniza Frias, ${ }^{*}$ Christopher Hernández Cortés, ${ }^{\ddagger}$ Silvia del Carmen Carrillo Ramírez, \\ Luis Antonio García Hernández, ${ }^{\S}$ José Manuel Portela Ortiz, " Juan Gerardo Esponda Pradoll
}

Citar como: Teniza FE, Hernández CC, Carrillo RSC, García HLA, Portela OJM, Esponda PJG. Incidencia del delirium postoperatorio en pacientes que ingresan a la Unidad de Terapia Intensiva (UTI) del Hospital Ángeles Pedregal. Acta Med. 2021; 19 (1): 67-75. https://dx.doi.org/10.35366/98573

\section{Resumen}

Objetivo: Evaluar la incidencia de delirium postoperatorio en la Unidad de Terapia Intensiva del Hospital Ángeles Pedregal, así como identificar los factores de riesgo y el pronóstico de los pacientes con delirium postoperatorio. Material y métodos: Estudio de análisis de frecuencia en el periodo comprendido del 01 de enero de 2018 al 31 de diciembre de 2019. Para el análisis estadístico se emplearon medidas de tendencia central y de dispersión. Se buscó la presencia de los factores de riesgo para delirium por riesgo relativo con IC al 95\%. Para las asociaciones, se utilizaron las pruebas de $\chi^{2}$ y $U$ de Mann-Whitney según se requiriera; $p<0.05$ fue considerada estadísticamente significativa. Resultados y conclusiones: Nuestra muestra se conformó de 84 pacientes. La incidencia de delirium postoperatorio durante su estancia en la UTI-HAP fue de $21.4 \%$. La presencia de ventilación mecánica se asoció estadísticamente $\left(\chi^{2}: 21.212, p=0.00\right)$ con la presencia de delirium (riesgo relativo 16.46; IC 95\% 4.72-57.3). Sí existió una diferencia estadísticamente significativa entre delirium postoperatorio y días de estancia en la Unidad de Terapia Intensiva (U Mann-Whitney 166; $p=0.000$ ).

Palabras clave: Delirium, delirium postoperatorio, Unidad de Terapia Intensiva.

\section{Abstract}

Objective: To evaluate the incidence of postoperative delirium in the Intensive Care Unit of the Angeles Pedregal Hospital, as well as identify the risk factors and prognosis of patients with postoperative delirium. Material and methods: Frequency analysis study in the period from January 1, 2018 to December 31, 2019. For statistical analysis, measures of central tendency and dispersion were used. The presence of risk factors for delirium was sought by relative risk with $95 \%$ $\mathrm{Cl}$. For the statistical associations, the $\chi^{2}$ and Mann-Whitney $\mathrm{U}$ tests were used as required. $\mathrm{p}<0.05$ was considered statistically significant. Results and conclusions: Our sample was consisted of 84 patients. The incidence of postoperative delirium at the UTI-HAP was $21.4 \%$. The presence of mechanical ventilation was statistically associated $\left(\chi^{2}: 21.212\right.$, $p=0.00$ ) with the presence of delirium (relative risk $16.46 ; 95 \%$ $\mathrm{Cl}$ 4.72-57.3). There was a statistically significant difference between postoperative delirium and days of stay in the Intensive Care Unit (U Mann-Whitney 166; $p=0.000)$.

Keywords: Delirium, postoperative delirium, Intensive Care Unit.

\footnotetext{
* Médico Residente de Anestesiología. Facultad Mexicana de Medicina de la Universidad La Salle.

* Médico adscrito a la Unidad de Terapia Intensiva.

§ Médico Anestesiólogo.

" Jefe de Anestesiología.

$\|$ Jefe de la Unidad de Terapia Intensiva.
}

Correspondencia:

Dr. Eduardo Teniza Frías

Correo electrónico: dr.teniza.frias@gmail.com

Aceptado: 09-10-2020.

www.medigraphic.com/actamedica

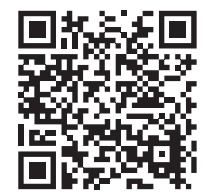




\section{INTRODUCCIÓN}

Las alteraciones en la neurocognición son una serie de fenómenos bien estudiados y descritos como entidades claramente establecidas, cuya relevancia radica en el aumento de costos económicos y humanos para un paciente. Dentro de este grupo encontramos al delirium: un estado agudo manifestado por fluctuaciones en el estado mental, alteración en la conciencia y la disminución o supresión total del nivel de atención.

\section{Definición de delirium postoperatorio (DPO)}

El DPO es aquel evento de delirium cuyo factor detonante es el evento anestésico quirúrgico. El término postoperatorio alude a toda la hospitalización posterior a la cirugía, siempre y cuando otras causas etiológicas de delirium hayan sido descartadas. ${ }^{2}$

Para mejorar su estudio, éste es dividido en dos grandes grupos: el delirium hiperactivo y el delirium hipoactivo. Este último se caracteriza por disminución de la actividad, estado de alerta, abstinencia, falta de conciencia y disminución del habla. Mientras que el delirium hiperactivo muestra agitación, actitud errante, irritabilidad y alucinaciones. ${ }^{3}$

Identificar el estado de delirium en cualquier paciente debe ser una tarea enfocada de manera intencionada, ya que el desarrollo de DPO es un factor que precipita un aumento en la morbimortalidad; además, una identificación temprana del mismo ayudará a resarcir de manera oportuna las consecuencias de éste. Mediante los criterios del Diagnostic and Statistical Manual of Mental Disorders, Fifth Edition (DSM-V) ${ }^{4}$ puede hacerse un diagnóstico de DPO, pero no siempre es aplicable al Área de Cuidados Críticos, por lo cual también puede ser complementado a partir del Confusion Assessment Method for the Intensive Care Unit (CAM ICU). ${ }^{5}$ Hayhurst plantea incluso al Richmond Agitation-Sedation Scale (RASS) ${ }^{6}$ como parte de un esquema ecléctico para la detección del DPO.

$$
\text { Factores de riesgo }
$$

Existen muchos factores de riesgo que predisponen a los pacientes a desarrollar DPO:

1. Daño cognitivo previo. Sin duda el principal factor que genera un riesgo. Es aquel paciente que previamente se encuentra con una alteración de las funciones mentales superiores, como lo son la demencia y enfermedad de Alzheimer. ${ }^{7}$ Esto ha sido planteado como consecuencia de que el individuo no presenta una reserva orgánica con la cual pueda hacer frente a un evento anestésico quirúrgico. $^{8}$
2. Edad. Conforme un paciente envejece éste se verá más predispuesto a ser afectado por el DPO debido a fenómenos degenerativos en el cerebro, los cuales sensibilizan a una fácil alteración de un frágil equilibrio que requiere para mantener un estado cognitivo íntegro. Esto ha sido mostrado en cirugía cardiaca y no cardiaca en las que existen series que muestran hasta $80 \%$ de desarrollo de DPO durante las primeras semanas del postanestésico e incluso llegan a $60 \%$ en un periodo tan largo como seis meses en pacientes mayores a 65 años. ${ }^{8}$

3. Sexo. Existen autores que correlacionan al sexo masculino como un factor de riesgo para el desarrollo de DPO con una razón de momios de 1.87, el resultado fue considerado después de realizar un metaanálisis de 17 estudios, en los cuales se consideró la incidencia de DPO en pacientes geriátricos de cirugía colorrectal. ${ }^{9}$ Cabe destacar que ésta no necesariamente tiene que ser una relación causal directamente atribuible al género masculino.

4. Tipo de cirugía. A través de diversas fuentes se ha planteado que el tipo de cirugía está estrechamente relacionado al desarrollo de DPO. Existen revisiones que muestran mayor incidencia a la cirugía genitourinaria, cardiológica, neurológica y a las intervenciones de urgencia al paciente hospitalizado. ${ }^{10}$ Durante un metaanálisis que contempló 24 ensayos clínicos y contemplaba 5,364 pacientes que fueron sometidos a artroplastia total de cadera, arrojó una razón de momios de 2.21 para desarrollo de DPO únicamente considerando el tipo de intervención quirúrgica. ${ }^{11}$

5. Nivel educativo. En una población con un nivel formativo alto, el cerebro está expuesto a actividades que podrían aumentar la eficacia de las sinapsis, por ello los pacientes con un acervo cultural más alto están en menor riesgo de desarrollar DPO. ${ }^{7}$ La mayoría de las revisiones, ya sean analíticas o descriptivas, concuerdan en que a partir de un capital cultural menor a un equivalente de educación media superior es suficiente para poder ser considerado como una población de riesgo ante el desarrollo de DPO, ${ }^{2}$ por dicha situación deben ser bien interrogados en medida de lo posible todos los pacientes que sean sometidos a cirugía.

Otros factores de riesgo también descritos son: fragilidad y comorbilidades importantes. ${ }^{1}$

El presente trabajo buscará la incidencia de delirium postoperatorio en pacientes que ingresan a la Unidad de Terapia Intensiva en las primeras 24 horas de su postoperatorio. Asimismo, se buscará identificar los factores de riesgo de los pacientes para el desarrollo del mismo. 


\section{MATERIAL Y MÉTODOS}

El estudio se desarrolló como análisis de frecuencia. Se incluyeron a todos los pacientes mayores de 18 años quienes fueron intervenidos quirúrgicamente e ingresaron en las primeras 24 horas del postoperatorio a la Unidad de Terapia Intensiva del Hospital Ángeles Pedregal, en el periodo comprendido del 01 de enero de 2018 al 31 de diciembre de 2019. Se excluyeron a todos los pacientes que permanecieron en la Unidad de Terapia Intensiva por menos de 24 horas, aquellos que fueron admitidos a la Unidad de Cuidados Coronarios, quienes no concluyeron su estancia en la unidad antes del 01 de enero de 2020 y aquellos quienes fueron trasladados a la Unidad de Terapia Intensiva como parte de una estrategia administrativa de optimización de espacio. Se eliminaron aquellos pacientes a quienes al momento de la recolección de datos fueron considerados como incompletos por el equipo de investigación.

Posteriormente, se prosiguió a la recolección de datos, mismos que se plasmaron en la hoja de recolección previamente establecida, que se dividió en cuatro partes:

Primera parte. Datos demográficos del paciente. Edad, género, calificación SOFA al ingreso, calificación SAPS al ingreso, condición de ingreso (A: falla orgánica, B: riesgo de falla orgánica y $\mathrm{C}$ : cuidados especiales).

Segunda parte. Diagnóstico de delirium postoperatorio. Para el diagnóstico de delirium utilizamos como base los criterios establecido en el DSM-V ${ }^{4}$ y el CAM (Confusion Assessment Method). ${ }^{5}$

Respecto al DSM-V, se requirió de por los menos tres criterios de los siguientes:

1. Alteración de la atención (por ejemplo, capacidad reducida para dirigir, centrar, mantener o desviar la atención) y de la conciencia (orientación reducida al entorno).

2. Alteración que aparece al poco tiempo (habitualmente horas o pocos días) constituye un cambio respecto a la atención y conciencia inicial y su gravedad atiende a fluctuar a lo largo del día.

3. Alteración cognitiva adicional (déficit de memoria, orientación, lenguaje o capacidad visoespacial o de la percepción).

4. Las alteraciones de los criterios 1 y 3 no se explican mejor por otra alteración neurocognitiva preexistente, debido a un consumo de drogas o medicamento, o exposición a toxinas.

Con respecto al CAM se estableció lo siguiente (mínimo tres criterios):
1. Cambios en el estado mental de inicio agudo y curso fluctuante.

2. Atención disminuida.

3. Pensamiento desorganizado.

4. Alteraciones a nivel de conciencia.

Para evitar el sesgo observador se realizó una capacitación del personal involucrado en el estudio que consistió en dos fases: la primera fase consistió en una clase teórica-práctica que incluyó ejemplos para el diagnóstico respecto a lo establecido en los criterios del DSM-V y CAM. La segunda fase consistió en un consenso del diagnóstico del tipo "double-check". Este consistió en la integración del diagnóstico posterior al ingreso a la Unidad de Terapia Intensiva por medio del médico residente en guardia siguiendo los criterios establecidos por el DSM-V y CAM (first-checkpoint). Posteriormente, la integración del diagnóstico era comprobado por los mismos instrumentos por el médico adscrito a cargo (second-checkpoint). Se definió como "paciente con delirium" únicamente a aquellos pacientes que cumplían con los criterios establecidos tanto por el DSM-V como por el CAM.

Tercera parte. Identificación de los factores de riesgo. Se contempló identificar después del proceso estadístico las características de la población que fuesen significativas para el desarrollo de delirio postoperatorio en nuestra unidad hospitalaria.

En ésta se incluyó los días de estancia en la Unidad de Terapia Intensiva y la mortalidad presentada.

Se utilizó la estadística con medidas de tendencia central y de dispersión, utilizando la prueba estadística Shapiro-Wilk para establecer la normalidad de cada una de nuestras variables cuantitativas. Al seguir lo plasmado en el marco teórico se buscó la presencia de los factores de riesgo para delirium por medio del cálculo del riesgo relativo con sus intervalos de confianza al 95\%. Los factores de riesgo investigados fueron: daño cognitivo previo, sexo masculino, presencia de comorbilidades, nivel educativo del paciente, tipo de cirugía realizada, tipo de anestesia administrada, si la cirugía fue de urgencia y la presencia de ventilación mecánica durante su estancia en la Unidad de Terapia Intensiva. Por último, para identificar la presencia de asociaciones se utilizaron las pruebas de $\chi^{2}$ y $U$ de MannWhitney según se requiriera, considerando una $p<0.05$ como estadísticamente significativa. El análisis estadístico en su totalidad fue realizado con el programa SPSS v. 19.

Cuarta parte. Pronóstico. Asimismo, se buscó encontrar si existió una diferencia significativa en la mortalidad y en los días que los pacientes permanecieron en la Unidad de Terapia Intensiva entre los pacientes que sí desarrollaron delirium y los que no. Esto se muestra en la Tabla 3. Como se puede observar, sí existió una diferencia estadísticamente significativa entre deli- 
rium postoperatorio y días de estancia en la Unidad de Terapia Intensiva (U Mann-Whitney 166; $p=0.000)$. Mientras que no se encontró una asociación estadísticamente significativa entre mortalidad y presencia de delirium $\left(\chi^{2} 3.782, p=0.115\right)$.

\section{RESULTADOS}

\section{Primera parte. Análisis demográfico (Tabla 1)}

Siguiendo los criterios de inclusión previamente establecidos, nuestra muestra se conformó de 84 pacientes (Figura
1). De éstos, $54.8 \%(n=46)$ fueron del sexo femenino y $45.2 \%(n=38)$ del sexo masculino, con una media (Shapiro-Wilk 0.978; $p=0.152$ ) de edad de 59 años (DE \pm 19 años) con un mínimo de edad de 18 años y un máximo de 98 (rango 80 años). Con respecto a las comorbilidades presentadas por los pacientes encontramos: hipertensión arterial sistémica (HAS) en 31\% $(n=26)$, diabetes mellitus 2 en $82.1 \%(n=69)$, hipotiroidismo en $9.5 \%(n=8)$, enfermedad pulmonar obstructiva crónica (EPOC) en $3.6 \%(n=3)$ y enfermedad renal crónica en $1.2 \%(n=1)$.






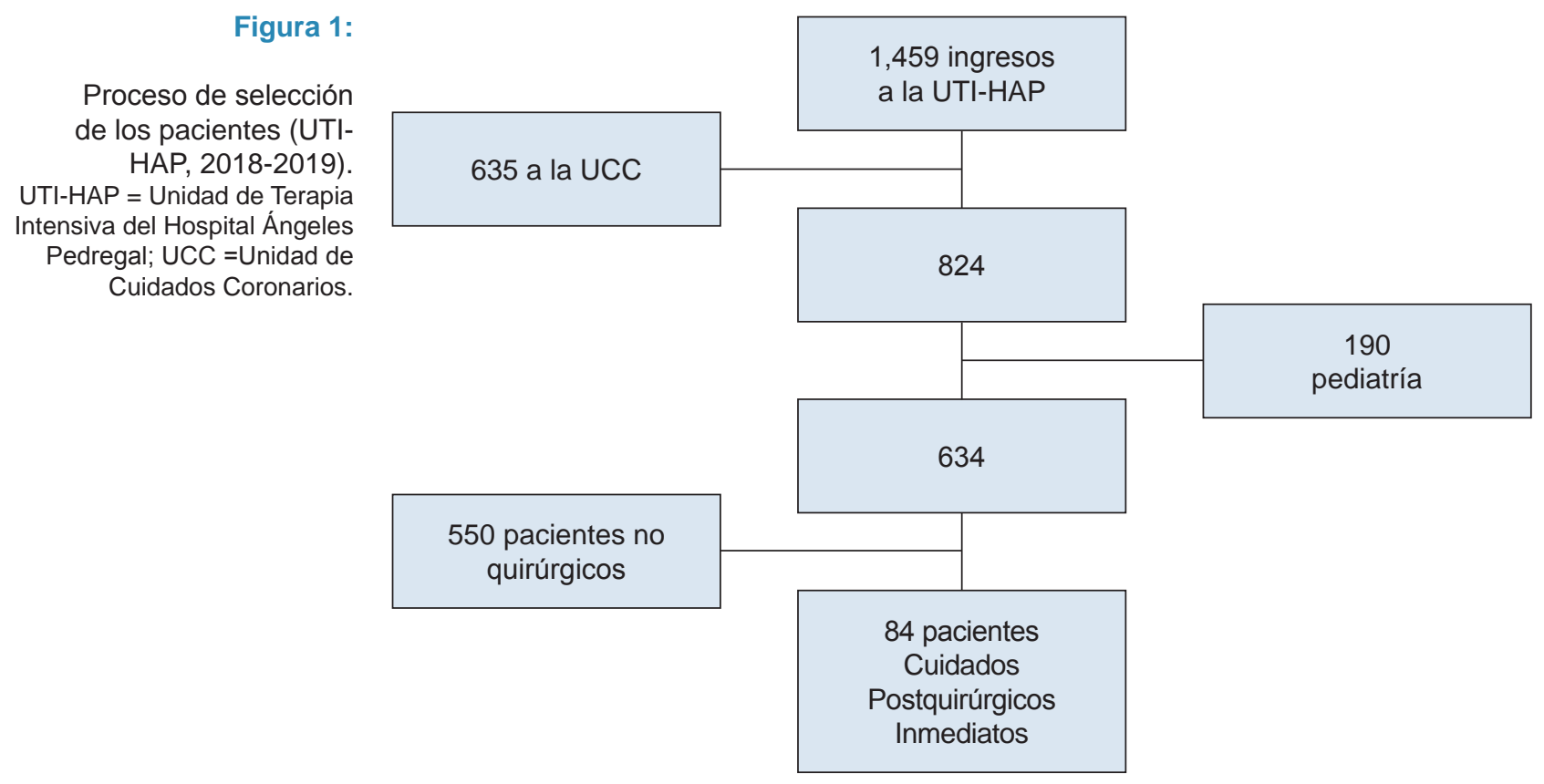

A su ingreso, la mediana de calificación SOFA presentada por los pacientes (Shapiro-Wilk 0.864; $p=0.00$ ) fue de 3 puntos (percentil 25 de 2 puntos y percentil 75 de 6 puntos) con una puntuación mínima de 0 y un máximo de 16 puntos (rango: 16). Mientras que la probabilidad de mortalidad a su ingreso medido por la escala pronóstica SAPS 3 presentó una mediana (Shapiro-Wilk 0.864; $\mathrm{p}=$ 0.00 ) de $22.4 \%$ (percentil 25 del $5 \%$ y percentil 75 de $37 \%$ ) con una probabilidad mínima de $1 \%$ y un máximo de $88 \%$ (rango: 87 ).

La frecuencia de acuerdo con el tipo de cirugía de los pacientes que ingresaron a la Unidad de Terapia Intensiva fue la siguiente: $47.6 \%(n=40)$ fueron de cirugía general, $45.2 \%(n=38)$ de neurocirugía, $3.6 \%$ $(n=3)$ de ginecología y obstetricia, $1.2 \%(n=1)$ de ortopedia, cirugía vascular y endoscopia cada uno. El $35.7 \%(n=30)$ de las cirugías fueron clasificadas como cirugías de urgencia.

Asimismo, a 71.4\% ( $\mathrm{n}=60)$ de los pacientes se les administró anestesia general, mientras que a 26.2\% ( $n=$ $22)$ anestesia total endovenosa y a $2.4 \%(n=2)$ algún tipo de anestesia neuroaxial.

La mortalidad presentada en nuestros pacientes fue de $3.6 \%(n=3)$. El 26.2\% $(n=22)$ requirió de ventilación mecánica en algún momento durante su estancia en la Unidad de Terapia Intensiva. De éstos, uno requirió de ventilación mecánica no invasiva, mientras que el resto (21) requirió de ventilación mecánica invasiva. La muestra presentó una mediana (Shapiro-Wilk 0.503; $p=0.00$ ) de 0 días de ventilación mecánica invasiva (percentil 25 de 0 días, percentil 75 de 1 día), con un mínimo de cero y un máximo de 14 días (rango 14 días).

Por otro lado, presentaron una mediana (Shapiro-Wilk 0.669; $p=0.00$ ) de dos días de estancia en la Unidad de Terapia Intensiva (percentil 25 de 1 día, percentil 75 de 4 días), con un mínimo de un día de estancia y un máximo de 19 días (rango: 18 días).

\section{Segunda parte. Delirium}

La incidencia de pacientes que desarrollaron delirium durante su estancia en la Unidad de Terapia Intensiva posterior a un procedimiento quirúrgico fue de $21.4 \%$ $(n=18)$.

\section{Análisis descriptivo de los pacientes con delirium}

De los 18 pacientes que presentaron delirium, 10 (55.6\%) fueron del sexo femenino y ocho (44.4\%) fueron del sexo masculino, con una media (Shapiro-Wilk 0.947; $p=0.375$ ) de edad de 57 años ( $D E \pm 18$ años) con un mínimo de edad de 18 años y un máximo de 93 (rango 75 años). Con una media (Shapiro-Wilk 0.930; $p=0.194$ ) de calificación SOFA a su ingreso de 3.7 puntos ( $\mathrm{DE} \pm 2.74$ ), con una puntuación mínima de cero y un máximo de 11 puntos (rango: 11). Mientras que la probabilidad de mortalidad a su ingreso medido por la escala pronóstica SAPS 3 presentó una media (Shapiro-Wilk 0.943; $p=0.324$ ) de 43.5\% (percentil 25 del $28.5 \%$ y percentil 75 de $49.25 \%$ ) con una probabilidad mínima de 1\% y un máximo de 78\% (rango: 
77). La mayoría de los pacientes fueron postoperados de cirugía general (nueve; 50\%), seguidos de neurocirugía (siete; 38.9\%). Asimismo, seis (33.3\%) fueron considerados cirugía de urgencia. De acuerdo con el tipo de anestesia administrada en los pacientes con delirium, a 94.4\% ( $n=$ 17) se le administró anestesia general balanceada y a $5.6 \%$ $(n=1)$ anestesia total endovenosa.

Trece $(72.2 \%)$ de los pacientes con delirium requirieron de ventilación mecánica durante su estancia en la Unidad de Terapia Intensiva, con una mediana (Shapiro-Wilk $0.889 ; p=0.03$ ) de 0 días (percentil 25 de 0 , percentil
75 de 8 días). Mientras que la media (Shapiro-Wilk 0.942; $\mathrm{p}=0.307$ ) de días de estancia en la UTI fue de 2.15 (DE \pm 1.47 ). Por último, dos (66.6\%) de los tres pacientes que fallecieron fueron diagnosticados con delirium durante su estancia en la Unidad.

\section{Tercera parte. Identificación de los factores de riesgo}

Se buscó identificar los factores de riesgo para presentar delirium (Tabla 2). Únicamente la presencia de ventilación mecánica $\chi^{2} 21.212, \mathrm{p}=0.00$; riesgo relativo de 16.46,

Tabla 2: Análisis de los factores de riesgo para el desarrollo de delirium (UTI-HAP, 2018-2019).

\begin{tabular}{|c|c|c|c|c|c|c|}
\hline & Delirium & No delirium & $\chi^{2}$ & $\mathrm{p}$ & RR (IC 95\%) & Sign. \\
\hline Daño cognitivo previo & 5 & 7 & 3.406 & 0.120 & $0.308(0.084-1.127)$ & \\
\hline Edad > a 60 años & 12 & 30 & 2.545 & 0.183 & $2.40(0.804-7.161)$ & \\
\hline Sexo masculino & 8 & 30 & 0.006 & 1.00 & $1.042(0.365-2.972)$ & \\
\hline Comorbilidades & & & & & & Sí \\
\hline HAS & 4 & 22 & 0.817 & 0.566 & $0.571(0.168-1.942)$ & \\
\hline DM & 7 & 8 & 6.908 & 0.015 & $4.614(1.387-15.346)$ & \\
\hline ERC & 1 & 0 & 3.711 & 0.054 & NA & \\
\hline EPOC & 1 & 2 & 0.262 & 0.609 & NA & \\
\hline HIP & 1 & 7 & 0.419 & 1.000 & NA & \\
\hline $\begin{array}{l}\text { Presencia de dos o más } \\
\text { comorbilidades }\end{array}$ & 5 & 10 & 1.537 & 0.215 & $2.154(0.629-7.380)$ & \\
\hline $\begin{array}{l}\text { Nivel educativo del } \\
\text { paciente }\end{array}$ & & & 3.177 & 0.365 & NA & \\
\hline Primaria & 0 & 1 & & & & \\
\hline Secundaria & 3 & 4 & & & & \\
\hline Bachillerato & 1 & 10 & & & & \\
\hline Licenciatura & 14 & 51 & & & & \\
\hline $\begin{array}{l}\text { Educación: preparatoria o } \\
\text { licenciatura }\end{array}$ & 15 & 61 & 1.356 & 0.360 & $0.410(0.088-1.909)$ & \\
\hline Tipo de cirugía & & & 8.656 & 0.124 & NA & \\
\hline Neurocirugía & 7 & 31 & & & & \\
\hline CG & 9 & 31 & & & & \\
\hline Endos & 1 & 0 & & & & \\
\hline TyO & 1 & 0 & & & & \\
\hline GyO & 0 & 3 & & & & \\
\hline Vasc & 0 & 1 & & & & \\
\hline Tipo de anestesia & & & 5.969 & 0.51 & NA & \\
\hline $\begin{array}{l}\text { Anestesia general } \\
\text { balanceada }\end{array}$ & 17 & 43 & & & & \\
\hline TIVA & 1 & 21 & & & & \\
\hline Neuroaxial & 0 & 2 & & & & \\
\hline Cirugía de urgencia & 6 & 24 & 0.57 & 1.00 & $0.875(0.291-2.631)$ & \\
\hline Ventilación mecánica & 13 & 9 & 21.212 & 0.000 & $12.667(3.792-42.311)$ & Sí \\
\hline
\end{tabular}

UTI-HAP = Unidad de Terapia Intensiva del Hospital Ángeles Pedregal; RR = riesgo relativo; Sign. = significativo; HAS = hipertensión arterial sistémica; DM = diabetes mellitus; $\mathrm{ERC}$ = enfermedad renal crónica; $\mathrm{EPOC}$ = enfermedad pulmonar crónica obstructiva; HIP = hipotiroidismo; NA = no aplica; CG = cirugía general; Endos = endoscopia; TyO = Trauma y Ortopedia; GyO = Ginecología y Obstetricia; Vasc = cirugía vascular; TIVA = anestesia total endovenosa. 
Tabla 3: Mortalidad y dias de estancia en pacientes con delirium (UTI-HAP, 2018-2019).

\begin{tabular}{lcccc} 
& Delirium & No delirium & U Mann-Whitney/ $\chi^{2}$ & $p$ \\
\hline Mortalidad & 2 & 1 & 3.782 & 0.115 \\
Días de estancia en la UTI-HAP & 66.28 & 36.02 & 166 & 0.000 \\
\hline UTI-HAP = Unidad de Terapia Intensiva del Hospital Ángeles Pedregal. & &
\end{tabular}

IC 95\% 4.72-57.3) y la presencia de diabetes mellitus $\left(\chi^{2}\right.$ $6.908, p=0.015$; riesgo relativo de 4.19 , IC 95\% 1.38715.346) se asociaron estadísticamente con la presencia de delirium.

\section{DISCUSIÓN}

Los pacientes mayores de 65 años reciben más de 1/3 de los más de los 40 millones de procedimientos anestésicos administrados anualmente en los Estados Unidos. ${ }^{3}$ Por lo tanto, es cada vez más importante que los médicos perioperatorios que atienden a pacientes geriátricos comprendan el complejo síndrome del delirium postoperatorio.

La incidencia de delirium postoperatorio en nuestra Unidad de Terapia Intensiva fue de $21.4 \%$. Hayhurst y colaboradores $^{6}$ refirieron una incidencia de $45 \%$ de los pacientes que cursan su estancia postquirúrgica inmediata en el Área de Cuidados Postquirúrgicos, alcanzando cifras de hasta $50 \%$ de los pacientes que ya están en el área de hospitalización. Por otro lado, Schenning y colaboradores ${ }^{3}$ refirieron que en poblaciones de alto riesgo el DPO puede alcanzar hasta $70 \%$ de incidencia, aunque esta información puede llegar a variar según las pruebas diagnósticas y el tipo de cirugía realizada.

La mayor implicación ante el desarrollo de DPO es la mortalidad, ya que en reportes de Europa la tasa de mortalidad hospitalaria es de 3\% después de la cirugía electiva y casi de $10 \%$ después de una cirugía de emergencia. ${ }^{12}$

Mediante nuestro estudio logramos dilucidar que la mortalidad presentada en nuestra Unidad de Terapia Intensiva no se vio afectada de manera estadísticamente significativa por el desarrollo de DPO; sin embargo, sí demostramos que aumenta el número de días de estancia en nuestra Unidad de Terapia Intensiva.

Otros factores perioperatorios que pueden afectar, o empeorar el DPO, y que no se investigaron en nuestro estudio incluyen: dolor, ciertos medicamentos, impedimentos sensoriales, inmovilización, falta de sueño, deshidratación y anomalías específicas de laboratorio (niveles séricos de sodio, niveles de glucosa, fracción de eyección, creatini- na o concentraciones de hemoglobina). ${ }^{13}$ En pacientes hospitalizados por enfermedad crítica se han demostrado deficiencias cognitivas y funcionales más marcadas a largo plazo. ${ }^{14,15} \mathrm{Al}$ señalar dicha idea también se comprende el motivo que subyace al desarrollo de DPO con mayor incidencia en las unidades de terapia intensiva, ya que las características demográficas que presentan estos pacientes son, en muchos casos, las condiciones ideales para presentar DPO.

Bajo las consideraciones anestésicas, en la evidencia en la literatura se pueden encontrar datos que no tienen la fuerza suficiente como para poder hacer una recomendación grado IA para sopesar más una técnica anestésica, ya sea general o regional, ya que al recibir anestesia neuroaxial no se observaron diferencias en el delirium postoperatorio en comparación con los que reciben anestesia general. ${ }^{16,17}$ Dentro de los resultados arrojados a través de nuestro estudio, tampoco se encontró una asociación estadísticamente significativa entre la presencia de una técnica anestésica general balanceada, endovenosa o regional y la presencia de delirium. Miller y colaboradores ${ }^{18}$ Ilevaron a cabo en 2018 un metaanálisis (28 ensayos clínicos; 4,507 pacientes), en el cual se compara la anestesia total endovenosa y la anestesia general balanceada para cirugía mayor no cardiaca. En éste se considera que la anestesia endovenosa basada en propofol sí puede disminuir la incidencia de DPO, pero como en muchos metaanálisis, esta información debería tomarse con cautela, ya que la heterogeneidad de los estudios se encuentra aclarada en dicho documento. ${ }^{18}$ Por nuestra parte, no encontramos una diferencia que pueda asociarse de manera significativa al desarrollo de delirium.

Los opioides han sido desde hace mucho tiempo una de las piedras angulares en la analgesia postoperatoria. ${ }^{19}$ La cantidad de opioide que se administra a un paciente durante el periodo perioperatorio se ha vinculado con el desarrollo de DPO, debido a que estos fármacos son capaces de alterar la recaptura de dopamina en el núcleo accumbens, ya que aumentan la liberación de dopamina a través de la activación de los receptores mu localizados 
en el área tegmental ventral. ${ }^{11}$ En específico, la morfina y la meperidina han sido los opioides con mayor asociación hacia el desarrollo de delirium. ${ }^{3}$ En este sentido, hay que considerar que dentro de nuestra unidad es administrado como sedoanalgesia en muchos pacientes que requieren ventilación mecánica invasiva, la cual es llevada a cabo mediante fentanilo o un derivado de los opioides en todos los pacientes; por ello es menester de los médicos considerar esto como un factor de riesgo, ya que los pacientes que necesitaron de ventilación mecánica invasiva durante su estancia en nuestra unidad sí se vieron mayormente afectados en cuestión de DPO, por lo que no podemos asegurar mediante este estudio si es la ventilación mecánica per se o la administración de los opioides fuertes el factor de riesgo para la presencia de delirium postoperatorio en nuestra unidad.

Por lo antes mencionado, muchos autores siguen buscando una estrategia que disminuya la incidencia de DPO. Khan administró haloperidol de forma profiláctica en 67 pacientes que fueron sometidos a cirugía no cardiaca, pero no mostró una diferencia significativa en la incidencia ni en la duración de los eventos. ${ }^{19}$

De manera similar, Khan, ${ }^{19}$ a través de su metaanálisis en el que contempla 10 ensayos clínicos aleatorizados, no pudo llegar a una conclusión que permitiera recomendar la administración de fármacos profilácticos para evitar el desarrollo de DPO.

La dexmedetomidina es un fármaco que actúa como un alfa dos agonista, el cual ha mostrado mejores resultados con respecto al desarrollo de DPO cuando se utiliza como sedación base en comparación con agentes hipnóticos que generan su efecto a través de los canales del ácido gamma amino butírico (GABA), como lo son el midazolam o el propofol, ${ }^{20}$ por las características inherentes de nuestro estudio no todos los pacientes que fueron contemplados para este análisis recibieron dosis de dicho fármaco, por lo cual sería interesante, en este sentido, futuras investigaciones dentro de nuestra unidad.

Para finalizar queremos recalcar que el DPO es una entidad multifactorial sumamente estudiada desde sus factores de riesgo, fisiopatología, prevención e impacto en el desenlace clínico de los pacientes, ya que el desarrollo de este fenómeno ha demostrado un enorme impacto en la morbimortalidad, días de estancia intrahospitalaria junto con costos médicos y económicos, por ello es de vital importancia considerar continuar el estudio del mismo.

\section{CONCLUSIONES}

La incidencia de delirium postoperatorio que ingresó a la Unidad de Terapia Intensiva del Hospital Ángeles
Pedregal fue de $21.4 \%$. La presencia de ventilación mecánica durante su estancia en la UTI-HAP aumenta de manera significativa el riesgo de presentar delirium postoperatorio. La presencia de DPO aumenta significativamente los días de estancia en la Unidad de Terapia Intensiva.

\section{REFERENCIAS}

1. Rengel KF, Pandharipande PP, Hughes CG. Postoperative delirium. Presse Med. 2018; 47 (4 Pt 2): e53-e64.

2. Evered L, Silbert B, Knopman DS, Scott DA, DeKosky ST, Rasmussen LS et al. Recommendations for the nomenclature of cognitive change associated with anaesthesia and surgery-2018. Br J Anaesth. 2018; 121 (5): 1005-1012.

3. Schenning KJ, Deiner SG. Postoperative delirium in the geriatric patient. Anesthesiol Clin. 2015; 33 (3): 505-516.

4. American Psychiatric Association. Diagnostic and statistical manual of mental disorders. 5th ed. Arlington, VA: American Psychiatric Association; 2015.

5. Carrillo R, Carrillo JR. Delirio en el enfermo grave. Rev Asoc Mex Med Crit y Ter Int. 2007; 21: 38-44.

6. Hayhurst CJ, Pandharipande PP, Hughes CG. Intensive Care Unit Delirium: a review of diagnosis, prevention, and treatment. Anesthesiology. 2016; 125 (6): 1229-1241.

7. Berger $\mathrm{M}$, Schenning $\mathrm{KJ}$, Brown $\mathrm{CH}$ 4th, Deiner SG, Whittington RA, Eckenhoff RG et al. Best practices for postoperative brain health: recommendations from the fifth International Perioperative Neurotoxicity Working Group. Anesth Analg. 2018; 127 (6): 14061413.

8. Kotekar N, Shenkar A, Nagaraj R. Postoperative cognitive dysfunction - current preventive strategies. Clin Interv Aging. 2018; 13: 22672273.

9. Yang Z, Wang XF, Yang LF, Fang C, Gu XK, Guo HW. Prevalence and risk factors for postoperative delirium in patients with colorectal carcinoma: a systematic review and meta-analysis. Int J Colorectal Dis. 2020; 35 (3): 547-557.

10. Banchs RJ, Lerman J. Preoperative anxiety management, emergence delirium, and postoperative behavior. Anesthesiol Clin. 2014; 32 (1): $1-23$.

11. Yang $Y$, Zhao X, Dong T, Yang Z, Zhang Q, Zhang Y. Risk factors for postoperative delirium following hip fracture repair in elderly patients: a systematic review and meta-analysis. Aging Clin Exp Res. 2017; 29 (2): 115-126.

12. Aldecoa C, Bettelli G, Bilotta F, Sanders RD, Audisio R, Borozdina A et al. European Society of Anaesthesiology evidence-based and consensus-based guideline on postoperative delirium. Eur J Anaesthesiol. 2017; 34 (4): 192-214.

13. Weinstein SM, Poultsides L, Baaklini LR, Mörwald EE, Cozowicz C, Saleh JN et al. Postoperative delirium in total knee and hip arthroplasty patients: a study of perioperative modifiable risk factors. Br J Anaesth. 2018; 120 (5): 999-1008.

14. Rengel KF, Hayhurst CJ, Pandharipande PP, Hughes CG. Long-term cognitive and functional impairments after critical illness. Anesth Analg. 2019; 128 (4): 772-780.

15. Eriksson LI, Lundholm C, Narasimhalu K, Sandin R, Jin YP, Gatz M et al. Hospitalization, surgery, and incident dementia. Alzheimers Dement. 2019; 15 (4): 534-542.

16. Silbert BS, Evered LA, Scott DA. Incidence of postoperative cognitive dysfunction after general or spinal anaesthesia for extracorporeal shock wave lithotripsy. Br J Anaesth. 2014; 113 (5): 784-791. 
17. Fong HK, Sands LP, Leung JM. The role of postoperative analgesia in delirium and cognitive decline in elderly patients: a systematic review. Anesth Analg. 2006; 102 (4): 1255-1266.

18. Miller D, Lewis SR, Pritchard MW, Schofield-Robinson OJ, Shelton CL, Alderson Petal. Intravenous versus inhalational maintenance of anaesthesia for postoperative cognitive outcomes in elderly people undergoing noncardiac surgery. Cochrane Database Syst Rev. 2018; 8 (8): CD012317.
19. Khan BA, Perkins AJ, Campbell NL, Gao S, Khan SH, Wang S et al. Preventing postoperative delirium after major noncardiac thoracic surgery-a randomized clinical trial. J Am Geriatr Soc. 2018; 66 (12): 2289-2297.

20. Vlisides P, Avidan M. Recent advances in preventing and managing postoperative delirium. F1000Res. 2019; 8: F1000 Faculty Rev607. 